Arq. Bras. Med. Vet.Zootec., v.56, n.5, p.618-622, 2004

\title{
Prótese coxofemoral em cães: Relato de dois casos
}

\author{
[Coxofemoral prosthesis in dogs: two cases] \\ S.A. Arias S. $^{1}$, C.M.F. Rezende ${ }^{2 *}$, A. Alvarez ${ }^{3}$, M.V. Souza ${ }^{4}$ \\ ${ }^{1}$ Doutorando em Ciência Animal - Universidade Federal de Minas Gerais \\ ${ }^{2}$ Escola de Veterinária - Universidade Federal de Minas Gerais \\ Caixa Postal 567 \\ 30123-970 - Belo Horizonte, MG \\ ${ }^{3}$ Escola de Veterinária - Universidade de Buenos Aires, Argentina \\ ${ }^{4}$ Mestrando em Clínica e Cirurgia Veterinárias - Universidade Federal de Minas Gerais
}

\section{RESUMO}

Foram realizadas duas cirurgias de prótese total da articulação coxofemoral em cão. Em um caso observou-se luxação caudal da prótese 72 horas após a cirurgia, que foi reduzida pelo método fechado, mantendo-se bandagem de Ehmer por 15 dias.O segundo caso evoluiu favoravelmente sem complicações pós-operatórias.Os dois pacientes foram avaliados periodicamente por 18 meses, mostrando desempenho clínico satisfatório, o que mostra a eficiência da técnica.

Palavras-chave: cão, displasia coxofemoral, cirurgia, prótese total coxofemoral, osteoartrose

\begin{abstract}
Two canine total hip replacements were performed. In one case, caudal luxation was observed 72 hours after the surgery, and it was treated by the closed reduction method, maintaining the Ehmer's sling for 15 days. The second case evoluted favourably without any postsurgical complication. Both patients were periodically evaluated during the 18 months that followed the surgery, showing a satisfactory clinical performance, that suggests the efficacy of the surgical technique.
\end{abstract}

Keywords: dog, hip dysplasia, surgery, total hip prostheses, osteoarthroses

\section{INTRODUÇÃO}

As lesões incapacitantes temporárias ou permanentes que acometem a articulação coxofemoral canina são freqüentes (Massat, 1995). No cão atleta, os principais problemas são decorrentes de trauma externo, ocasionando luxações de cabeça femoral, fraturas acetabulares e fraturas de cabeça e/ou colo femoral. Outras lesões observadas são a displasia coxofemoral e as decorrentes de treinamento (Wendelburg, 1998). No entanto, na prática ortopédica

Recebido para publicação em 2 de julho de 2003

Recebido para publicação, após modificações, em 26 de novembro de 2003

*Autor para correspondência

E-mail: cleuza@dedalus.lcc.ufmg.br veterinária a displasia coxofemoral tem sido a alteração mais comum em diversas raças, correspondendo aproximadamente a $25 \%$ dos casos (Richardson, 1992). Oitenta e dois por cento dos pacientes submetidos à prótese total da articulação, o são devido à displasia coxofemoral (Montgomery et al., 1992). Essa afecção é caracterizada pela instabilidade da articulação em animais imaturos, que resulta em má articulação dos componentes articulares e conseqüentemente no desencadeamento da doença articular degenerativa (Manley, 1993). 
Os principais procedimentos cirúrgicos utilizados para o tratamento dessa alteração são osteotomia pélvica tríplice, osteotomia intertrocantérica, excisão artroplástica de cabeça e colo femorais e a prótese total da articulação (Fossum et al., 1997). O objetivo deste trabalho é relatar dois casos de prótese total cimentada da articulação coxofemoral.

\section{CASUÍSTICA}

Caso $n^{\circ} 1$ (experimental) - Foi selecionado um animal, sem raça definida, no canil da prefeitura de Belo Horizonte, com $20 \mathrm{~kg}$ de peso. Realizouse radiografia ventro-dorsal da articulação coxofemoral, verificando-se ausência de alterações osteo-articulares. O animal foi preparado para cirurgia ortopédica empregando-se a abordagem e técnica cirúrgica descritas por Olmstead
(1987). Após preparação, foram implantados a prótese de aço inoxidável cimentada não modular (modelo Richards II), de tamanho médio $(8 \mathrm{~mm}$ de diâmetro) e componente acetabular de polietileno de ultra-alto peso molecular com diâmetro interno de $17 \mathrm{~mm}$ e externo de $25 \mathrm{~mm}$. Foi feita profilaxia antibiótica trinta minutos antes da cirurgia com cefalotina sódica via intravenosa na dose de $30 \mathrm{mg} / \mathrm{kg}$ e repetida 90 minutos após a primeira aplicação. A cefalotina foi continuada no pós-operatório a cada 12 horas até o resultado negativo de cultura bacteriana. Foi administrado cetoprofeno $1,0 \mathrm{mg} / \mathrm{kg}$ por três dias. O animal foi mantido no pós-operatório em canil individual com restrição dos movimentos.

Nas radiografias pós-operatórias observou-se posição varus da haste coxofemoral na incidência ventro-dorsal (Fig. 1a) e posição neutra na médio-lateral (Fig. 1b).

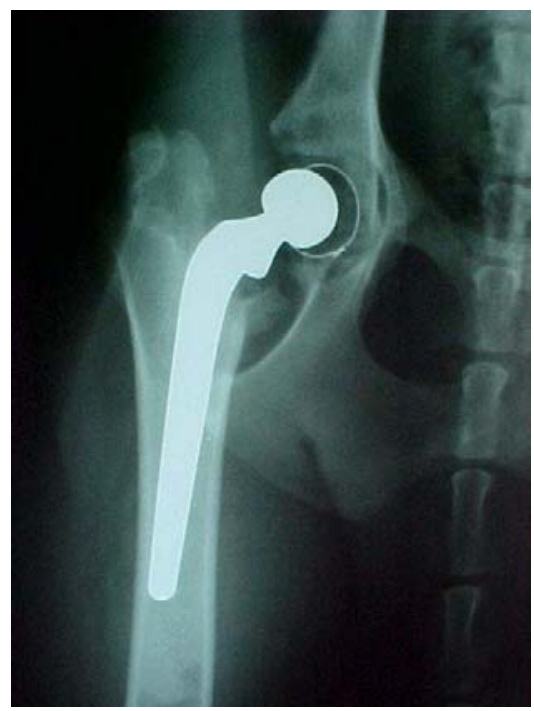

a

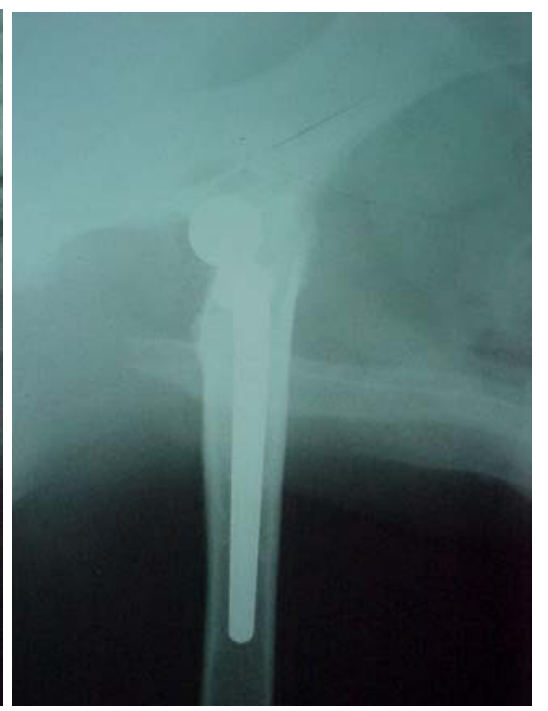

b

Figura 1. Incidências radiográficas da articulação coxofemoral: a- incidência ventro-dorsal logo após implante de prótese total coxofemoral (observa-se posição varus do implante); b- incidência médio-lateral pós-cirúrgica (observa-se posição neutra do implante).

No terceiro dia de pós-operatório, verificou-se claudicação acentuada sem apoio do membro. Foi realizada radiografia coxofemoral ventrodorsal que mostrou luxação caudo-dorsal do implante (Fig. 2a). No animal anestesiado foi realizada redução fechada da articulação protética, seguindo-se colocação de bandagem tipo Ehmer por 15 dias (Fig. 2b). 
Após a retirada da bandagem, o paciente mostrou acentuada hipotrofia muscular sem apoio do membro. Foi feita fisioterapia durante duas semanas, iniciando cada sessão com movimento passivo por 15 minutos, seguindo-se caminhada controlada em areia por 30 minutos. Após a fisioterapia o animal retornou à deambulação com apoio total do membro. Entretanto, foi evidenciada abdução na posição ortostática e em deambulação verificava-se acentuada rotação externa do membro, que reduziu para discreta com deambulação satisfatória após 18 meses. O paciente foi acompanhado mensalmente e foram feitas radiografias em intervalos de seis meses, evidenciando posição adequada do implante.

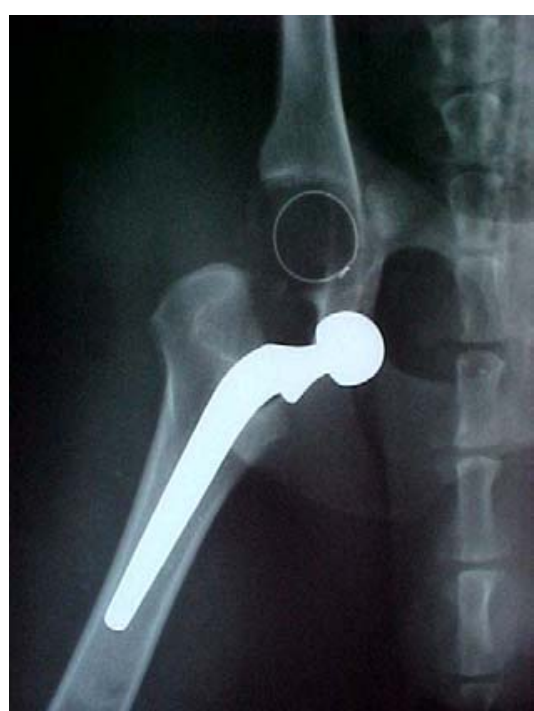

a

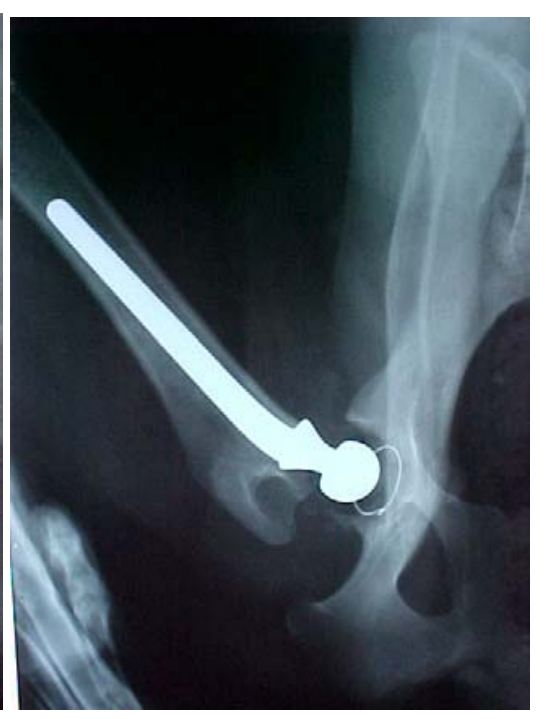

b

Figura 2. Incidências radiográficas da articulação coxofemoral: a- incidência ventro-dorsal (observa-se luxação caudo-dorsal do implante); b- incidência ventro-dorsal logo após redução fechada da luxação e bandagem de Ehmer.

Caso n. ${ }^{\circ} 2$ - Em julho de 2000 foi apresentado no hospital veterinário da Universidade Federal de Minas Gerais um cão da raça pastor alemão, macho, de oito meses de idade, com $31 \mathrm{~kg}$ de peso, que mostrava claudicação dos membros posteriores, mais acentuada no esquerdo, e andar bamboleante. Ao exame ortopédico verificou-se abdução dolorosa e instabilidade coxofemoral bilateral mais acentuada na articulação esquerda. A radiografia ventro-dorsal da pelve mostrou alterações ósseas compatíveis com displasia coxofemoral. As articulações mostravam subluxação, mais acentuada no membro esquerdo. Foi prescrito carprofeno ${ }^{1}, 75 \mathrm{mg}$ a cada 12 horas por 15 dias e dois comprimidos de sulfato de condroitina $^{2}$ diários por 30 dias. Após esse período, o cão apresentou vômito com

\footnotetext{
Rymadil, Pfizer

${ }^{2}$ Artroglycan, Syntex S. A.
}

permanência do desconforto à abdução coxofemoral. O sulfato de condroitina foi substituído por Osteorcart $70^{3}$, três comprimidos por 90 dias, ao final dos quais foi feito controle médico e constatada melhora no grau de claudicação. Também foi aconselhada a realização de fisioterapia.

Em março de 2001 foram realizadas novas radiografias, sendo observado agravamento da deformidade da cabeça e colo femorais e do acetábulo (Fig. 3a). Foi sugerida a substituição articular por prótese total. $\mathrm{O}$ animal foi preparado para cirurgia sob protocolo de cefalotina e cetoprofeno como descrito no caso anterior.

\footnotetext{
${ }^{3}$ Labyes, composição: D-L metionina 40mg, L-Cisteína 10mg, Arginina $\mathrm{HCl} 10 \mathrm{mg}$, Histidina $4 \mathrm{mg}$, Betaína $\mathrm{HCl} 4 \mathrm{mg}$, LGlutamina 16mg, Piridoxina $\mathrm{HCl} 4 \mathrm{mg}$, D-Glucosamina 16mg, Gluconato de $\mathrm{Cu} 10 \mathrm{mg}$, gluconato de $\mathrm{Mg} 20 \mathrm{mg}$, Gluconato de manganês $16 \mathrm{mg}$, Gluconato de Zinco $30 \mathrm{mg}$.
} 
Usou-se prótese modular de aço inoxidável cimentada $^{4}$ de tamanho médio $(8 \mathrm{~mm}$ de diâmetro) e componente acetabular de polietileno de ultra-alto peso molecular com diâmetro interno de $17 \mathrm{~mm}$ e externo de $27 \mathrm{~mm}$. Foi recomendado manutenção do cão no canil por 15 dias, seguindo-se restrição de exercícios por dois meses. Foram realizadas radiografias nas posições ventro-dorsal e médio-lateral sendo observado posição neutra do implante na incidência ventro-dorsal (Fig. 3b) e posição cranial com direcionamento caudal da ponta da haste coxofemoral na incidência médio lateral (Fig. 3c). O cão mostrou deambulação normal a partir do terceiro dia de pós-operatório com apoio completo do pé na posição de micção. No segundo e terceiro mês após a cirurgia o paciente podia correr, confirmando-se a resposta clínicocirúrgica satisfatória.

Ambos os pacientes foram novamente avaliados aos 18 meses após a cirurgia, evidenciando-se recuperação muscular, adequação dos tecidos à posição do implante e deambulação satisfatória no exame clínico ortopédico do primeiro caso. Atualmente esse paciente permanece como doador de sangue no hospital veterinário. O segundo caso mostrou desempenho físico satisfatório, com aumento aparente da massa muscular no membro operado, sugerindo maior suporte de peso neste e alivio da articulação displásica contra-lateral.

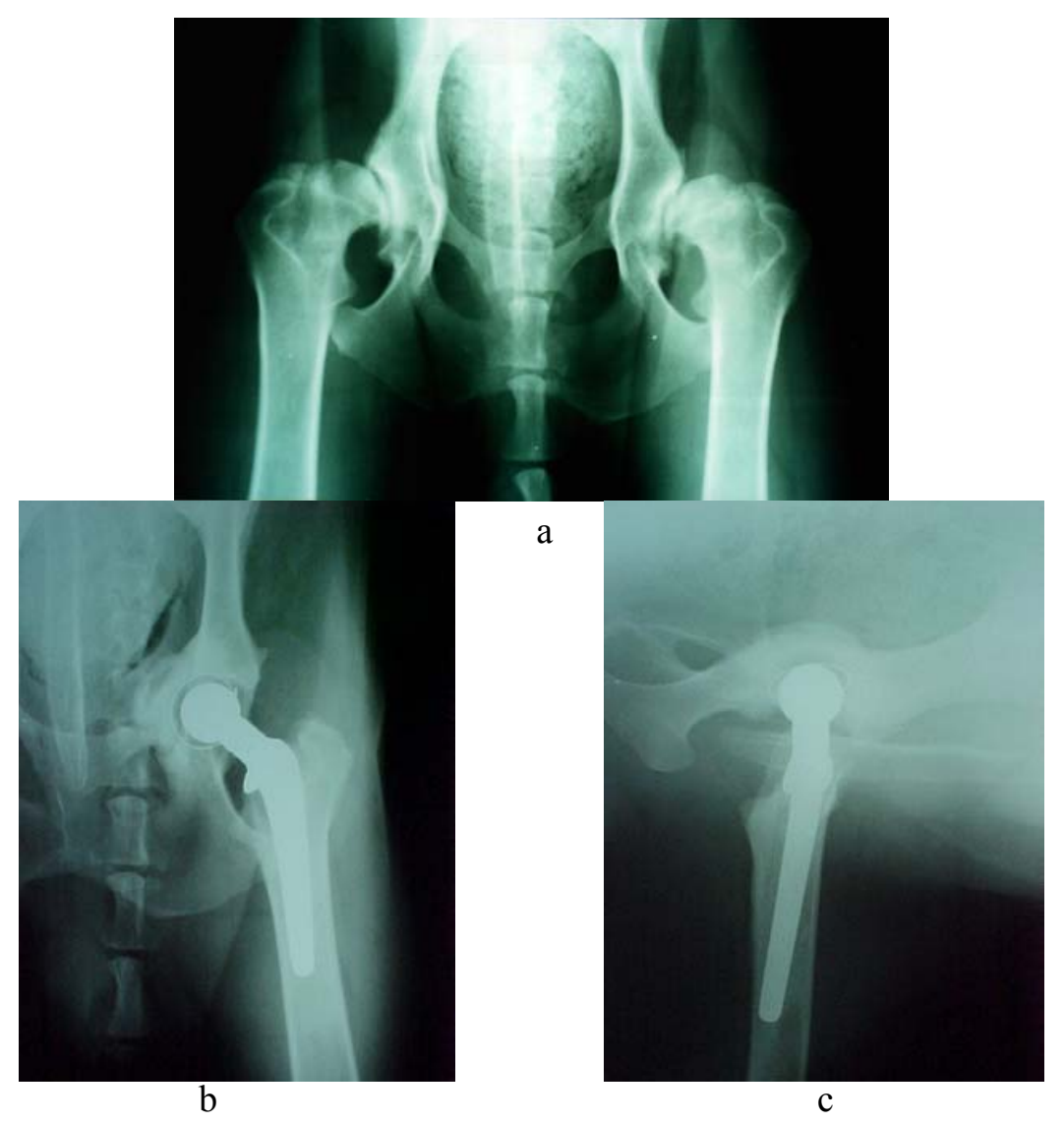

Figura 3. Incidências radiográficas da articulação coxofemoral: a- incidência radiográfica ventro-dorsal précirúrgica (observa-se acentuado grau de displasia coxo-femoral com sub-luxação); b- incidência ventro-dorsal logo após implante de prótese total coxofemoral (observa-se posição neutra do implante); c- incidência médiolateral pós-cirúrgica (observa-se posição cranial com inclinação caudal da ponta do implante).

${ }^{4}$ Implantes Fico, Buenos Aires, Argentina 


\section{DISCUSSÃO E CONCLUSÕES}

A maioria das complicações dessa técnica cirúrgica acontece aproximadamente até o quarto mês de pós-operatório (Olmstead et al., 1983), observando-se diminuição de sua incidência após 18 meses (Olmstead, 1987).

A luxação do implante é a complicação mais freqüentemente relatada no cão (Olmstead et al., 1983; Massat e Vasseur, 1994; Olmstead, 1995; Tomlinson e Mc Laughlin, 1996), observada no primeiro caso deste trabalho. A luxação pode ser atribuída a exercício precoce, inadequada orientação tanto do componente acetabular quanto do femoral e trauma externo (Montgomery et al., 1992). Nesse caso, provavelmente, decorreu de colocação e orientação inadequadas dos componentes femoral e acetabular. A colocação antevertida e com aumentado ângulo de abertura lateral do componente acetabular predispõem à luxação dorsal no pós-operatório imediato (Massat, 1995), como observado nesse paciente. A utilização de prótese não modular, cujo colo femoral é fixo e não pode ser ajustado à geometria do paciente no ato operatório, pode ter contribuído para essa complicação. Um colo protético muito longo pode explicar a abdução do membro (Dyce et al., 2000) observada no paciente.

Diferentes variáveis influenciam a posição do componente femoral como o nível de ostectomia do colo femoral, a remoção do bloco subtrocantérico, o direcionamento durante a inserção da haste e o diâmetro e tamanho da haste (Wylie et al., 1997; Schulz et al., 1998; Schulz, 2000). Os posicionamentos varus e inclinação caudal da ponta do implante são os mais freqüentemente verificados em cães como observado neste trabalho, porém são relacionados com afrouxamento do implante no homem (Schulz et al., 1998).

A prótese total coxofemoral é uma das técnicas mais efetivas no tratamento das alterações artríticas que acometem a articulação coxofemoral no cão, apresentando-se como a alternativa mais adequada em cães adultos com displasia coxofemoral.

\section{REFERÊNCIAS BIBLIOGRÁFICAS}

DYCE, J.; WISNER, E.R.; WANG, Q. et al. Evaluation of risk factors for luxation after total hip replacement in dogs. Vet. Surg., v.29, p.524-532, 2000.

FOSSUM, T.W.; HEDLUND, C.S.; HULSE, D.A. et al. Hip dysplasia: small animal surgery. Saint Louis: Mosby, 1997. P.942-949.

MANLEY, P.A. The hip joint. In: SLATTER, D. Textbook of small animal surgery. 2.ed. Philadelphia: Saunders, 1993. Cap.135, p.1786-1805.

MASSAT, B.J. Artroplastia cementada total de la cadera canina. Waltham Focus, v.5, p.21-31, 1995.

MASSAT, B.J.; VASSEUR, P.B. Clinical and radiographic results of total hip artroplasty in dogs: 96 cases (1986-1992). J. Am. Vet. Med. Assoc., v.205, p.448454, 1994.

MONTGOMERY, R.D.; MILTON, J.L.; PERNELL, R. et al. Total hip arthroplasty for treatment of canine hip dysplasia, Vet. Clin. North Am.: Small Anim. Pract., v.22, p.703-719, 1992.

OLMSTEAD, M.L. Canine cemented total hip replacements: state of the art. J. Small Anim. Pract., v.36, p.395-399, 1995

OLMSTEAD, M.L. Total hip replacement. Vet. Clin. North Am.: Small Anim. Pract., v.17, p.943-955, 1987.

OLMSTEAD, M.L.; HOHN, R.B.; TURNER, T.M. A five- year study of 221 total hip replacements in the dog. J. Am. Vet. Med. Assoc., v.183, p.191-194, 1983.

RICHARDSON, DC. The role of nutrition in canine hip dysplasia. Vet. Clin. North Am: Small Anim. Pract., v.22, p.529-540, 1992.

SCHULZ, K.S. Application of arthroplasty principles to canine cemented total hip replacement. Vet. Surg., v.29, p.578-593, 2000

SCHULZ, K.S.; VASSEUR, P.B.; STOVER, S.M. et al. Transverse plane evaluation of the effects of surgical technique on stem positioning and geometry of reconstruction in canine total hip replacement. Am. J. Vet Res., v.59, p.1071-1079, 1998.

TOMLINSON, J.; McLAUGHLIN, R. Total hip replacement: the best treatment for displastic dogs with osteoarthrosis. Vet. Med., v.91, p.118-124, 1996.

WENDELBURG, K.L. Disorders of the hip joint in the canine athlete._In: BLOOMBERG, M.S.; DEE, J.F.; TAYLOR, R.A. Canine sports medicine and surgery. Philadelphia: Saunders, 1998. Cap.21, p.174-195,

WYLIE, K.B.; DeYOUNG, D.J.; DROST, W.T. The effect of surgical approach on femoral stem position in canine cemented total hip replacement. Vet. Surg., v.26, p.62-66, 1997. 